\title{
Revisión de la literatura. \\ Manejo actual de la estenosis de carótida: Tratamiento endovascular comparado con endarterectomía
}

Drs. Elvira Jiménez G, Rafael Oteros F, Fernando Delgado A, Antonio Cano S.

Servicio de Radiología, Sección de Neurorradiología Diagnóstica y Terapéutica.

Hospital Universitario Reina Sofía, Córdoba. España.

Current management of carotid stenosis: Endovascular treatment versus endarterectomy

Abstract. Antiplatelet therapy used to be the only way to prevent further cerebrovascular events in patients with carotid stenosis. After publication of final results of the European Carotid Surgery Trial (ECST) and North American Symptomatic Carotid Endarterectomy Trial (NASCET), endarterectomy was considered the best treatment option. Currently, endovascular treatment of atherosclerotic carotid artery stenosis has been established as an alternative to surgical endarterectomy. Our aim is to show the evolution of endovascular therapy in carotid stenosis, to compare outcomes between endovascular, medical and surgical treatments, and to analize the current state of endovascular therapy. It is worth underscoring that many of the studies were conducted decades ago and consequently both the endovascular technique and medical treatments used then greatly differ from current practices. Several studies agree stenting benefits patients under 70 years of age and exhibits, at least, same short-term results as endarterectomy, along with higher rate of long-term restenosis. Indication for treatment in asymptomatic patients and the use of protection systems remain an unresolved issue. A multidisciplinary team should implement an individualized treatment plan for each patient.

Keywords: Angioplasty. Endarterectomy. Carotid stenosis. Stroke.

Resumen. En estenosis carotidea, la única manera de prevenir nuevos eventos cerebrovasculares era mediante antiagregación. Después de los estudios NASCET y ECST se estableció que la endarterectomía conseguía mejores resultados. Ahora se ha establecido la terapia endovascular como alternativa.

Nuestro objetivo es mostrar la evolución de la terapia endovascular en estenosis carotidea, contrastar los resultados entre tratamiento endovascular, médico y quirúrgico y exponer la situación actual de la terapia endovascular.

Algunos estudios se realizaron hace décadas y tanto la técnica endovascular como los tratamientos médicos, no son comparables con los de hoy. Varios estudios coinciden en que el stenting beneficia a menores de 70 años y presenta al menos iguales resultados que la endarterectomía a corto plazo, con mayor tasa de reestenosis a largo plazo. Quedan por resolver la indicación de tratamiento en pacientes asintomáticos o la utilización de sistemas de protección. El tratamiento debe ser individualizado en un equipo multidisciplinar.

Palabras clave: Angioplastía, Endarterectomia, Estenosis carotidea, Ictus carotideo.

Jiménez $E$, et al. Manejo actual de la estenosis de carótida: tratamiento endovascular comparado con endarterectomía. Rev Chil Radiol 2012; 18(2): 49-57.

Correspondencia: Dra. Elvira Jiménez Gómez / elvirajimenezgomez@gmail.com

Trabajo recibido el 14 de febrero de 2012, aceptado para publicación el 11 de junio de 2012.

\section{Introducción}

La estenosis arterioesclerótica de carótida interna es una de las causas más importantes de ictus isquémico. Por otro lado, el riesgo de ictus recurrente en pacientes con estenosis carotidea grave es de un $28 \%$ a los dos años ${ }^{(1,2)}$.

Dado que el riesgo que un paciente que ha sufrido un evento cerebrovascular, presente un nuevo evento,

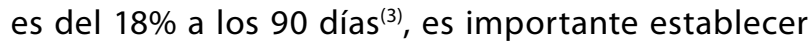
tratamientos que disminuyan este riesgo.

Previamente a la aparición de los tratamientos invasivos de la estenosis carotídea, la única manera de prevenir nuevos eventos era mediante la administración de antiagregantes, que conseguían una reducción moderada del riesgo de un nuevo evento (alrededor de un $4 \%$ a los dos años, según estudios). 
Así el tratamiento antiplaquetario demostró su utilidad en la prevención del ictus o de accidentes isquémicos transitorios ${ }^{(4)}$. Después de la publicación de los estudios NASCET y $\mathrm{ECST}^{(5,6)}$ se estableció que la endarterectomía conseguía mejores resultados, en el tratamiento de estenosis carotidea sintomática de más del $70 \%$ (siempre que la mortalidad perioperatoria no excediera el 6\%).

En la actualidad se ha establecido la terapia endovascular como alternativa a la endarterectomía en aquellos pacientes que se beneficiarían de la misma, pero en los que la cirugía no se considere adecuada, por presentar un alto riesgo quirúrgico o anestésico. El problema reside en la falta de consenso sobre criterios de indicación de la terapia endovascular, bien de tipo anatomicomorfológico relacionados con las características de la estenosis o de tipo clínico ${ }^{(7)}$.

El objetivo de la revisión es mostrar las evolución de la terapia endovascular para el tratamiento de la estenosis carotidea, en los últimos años, repasar los diversos estudios que se han realizado contrastando los resultados entre tratamiento endovascular, médico y quirúrgico y destacar cuál es la situación actual en relación con los criterios de indicación de terapia endovascular y qué problemas quedan aún por resolver.

\section{Angioplastía carotidea con balón}

La angioplastía con balón es una técnica en la que mediante un balón inflado a alta presión se realiza una dilatación de la estenosis. La primera angioplastía con balón para tratar una estenosis de carótida la realizó Kerber et al en $1980^{(8)}$.

Desde su aparición surgieron multitud de estudios que han intentado demostrar la no inferioridad de la misma con respecto a la endarterectomía.

Una de las series de seguimiento más prolongado es la de Gil-Peralta ${ }^{(9)}$ que en 1996 realizó 85 angioplastías con balón en 82 pacientes con estenosis carotidea sintomática igual o mayor al $70 \%$, y se siguieron durante 4 años. La tasa de éxito fue del $92 \%$ y la mortalidad a los 30 días fue del $0 \%$, con una tasa de morbilidad del $4,9 \%$. Esto mejoró los resultados del NASCET y ECST con la endarterectomía. La tasa de reestenosis fue del $6,7 \%$ durante un periodo de seguimiento medio de 18,7 meses (la tasa de reestenosis en la endarterectomía era del $10 \%$ durante el primer año de seguimiento). Todas esas reestenosis eran asintomáticas y se produjeron en su mayoría entre el tercer y el sexto mes postratamiento ${ }^{(10,11)}$.

A pesar de los buenos resultados iniciales de la angioplastía con balón, se observaron síntomas cardiovasculares por manipulación en el seno carotídeo, riesgo de disección intimal o desprendimiento de la placa de ateroma con subsiguiente embolización distal. Este es el problema más importante de esta técnica.

En los estudios realizados para examinar la frecuencia de embolias durante la angioplastía con balón mediante Doppler transcraneal, no se demostró correlación entre la frecuencia de embolismos y de secuelas neurológicas ni entre el número de émbolos detectados por el Doppler y la tasa de ictus postoperatorio ${ }^{(12-14)}$. Esto sirve para explicar la ausencia de correlación entre la detección de émbolos y la baja tasa de secuelas clínicas, ya que se cree que la mayoría de los émbolos detectados con el Doppler durante la angioplastía con balón son burbujas o microagregados menores de $200 \mu \mathrm{m}$ que se relacionan con una evolución favorable ${ }^{(12,13)}$. Otro de los problemas son los cuadros de isquemia cerebral secundaria ${ }^{(15)}$ a que durante el momento de inflado del balón disminuye el flujo sanguíneo cerebral.

Dada la tasa de reestenosis de la angioplastía con balón y la necesidad de retratamiento a un año, junto con los buenos resultados que dieron en cardiología la angioplastía con stent asistida con balón, surgieron las nuevas formas de tratamiento endovascular de la estenosis carótidea mediante angioplastía y colocación de stent en la zona de la estenosis.

\section{Angioplastía carotidea con stent}

Consiste en colocar en la zona de la estenosis un stent. En la mayoría de los casos se utilizan stents autoexpandibles, que no requieren balón para que se abran, solventando el problema de la disminución de flujo sanguíneo cerebral durante el inflado del balón.

Si queda estenosis residual, se puede asociar una dilatación con balón, previa o posterior a la colocación del stent. A su vez pueden utilizarse dispositivos de protección como filtros para atrapar las partículas que puedan soltarse y evitar embolismos distales. Las ventajas de la colocación del stent frente a la angioplastía sólo con balón son que evita en parte el desprendimiento de la placa, la disección intimal y presenta menos tasas de reestenosis tardías ${ }^{(15)}$.

Los primeros resultados de esta técnica derivan de estudios no aleatorizados, con un nivel de evidencia científica que no permitía una generalización del procedimiento ${ }^{(7)}$. Posteriormente surgieron estudios aleatorizados que analizaron la eficacia y seguridad del empleo de stent en comparación con la endarterectomía y compararon también la colocación de stent con y sin empleo de sistemas de protección. A continuación se enumeran los estudios más importantes al respecto. En la Tabla I se presentan de forma resumida los estudios más importantes sobre angioplastía vs endarterectomía.

Carotid and Vertebral Artery Transluminal Angioplasty Study (CAVATAS) ${ }^{(16)}$ : Es el primer estudio randomizado que compara el tratamiento endovascular (angioplastía y/o stent) frente al tratamiento quirúrgico con endarterectomía.

Incluyó a 504 pacientes; de los cuales el 90\% eran sintomáticos; sin demostrar diferencias en la morbimortalidad entre la angioplastía y cirugía (10 


\begin{tabular}{|c|c|c|c|c|c|}
\hline 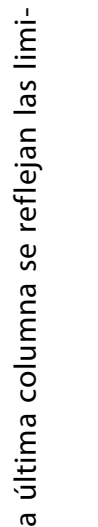 & 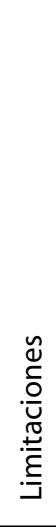 & 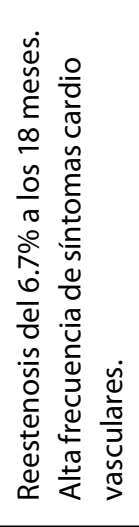 & & 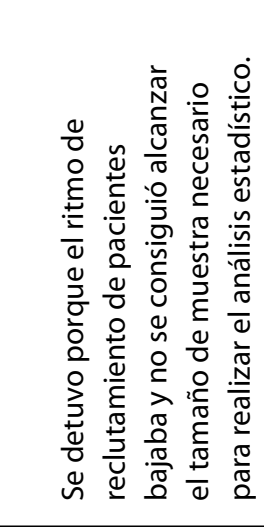 & 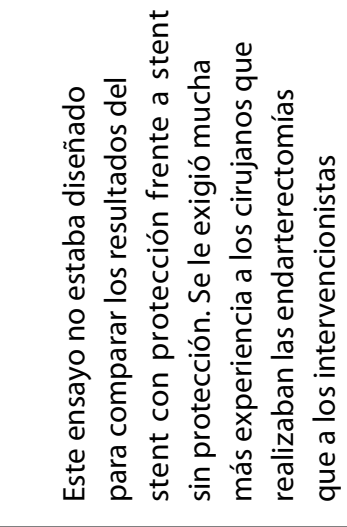 \\
\hline 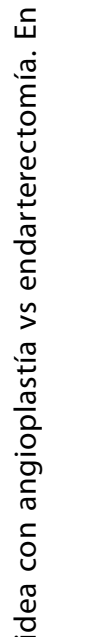 & $\begin{array}{l}\tilde{O} \\
\frac{0}{0} \\
\frac{\mathbb{J}}{5} \\
\widetilde{\mathscr{U}} \\
\end{array}$ & 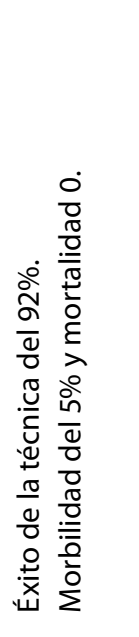 & 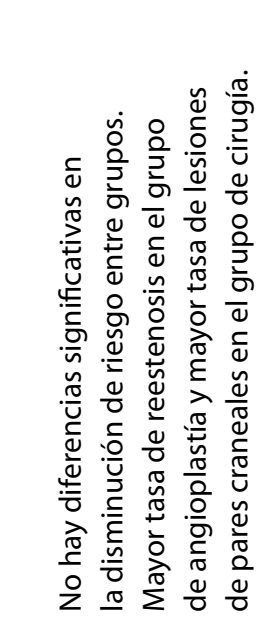 & 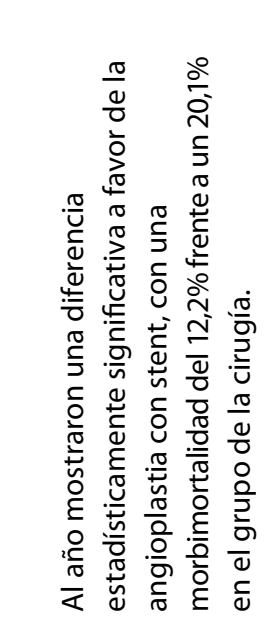 & 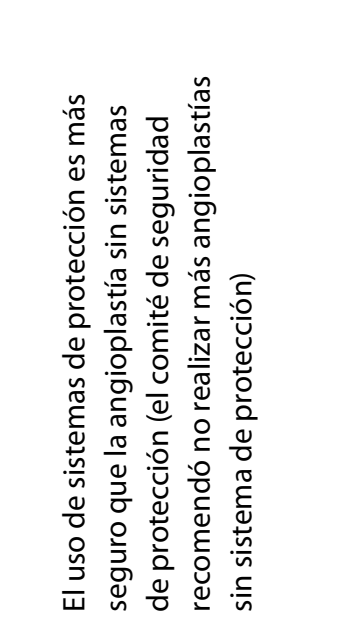 \\
\hline 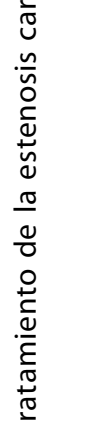 & 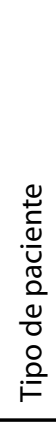 & 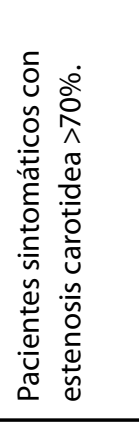 & 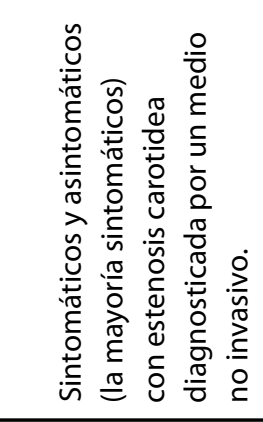 & 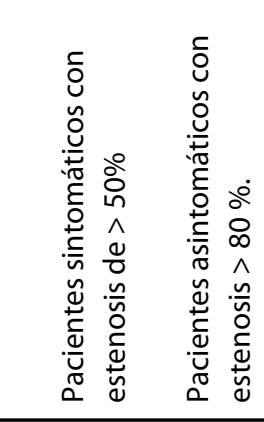 & 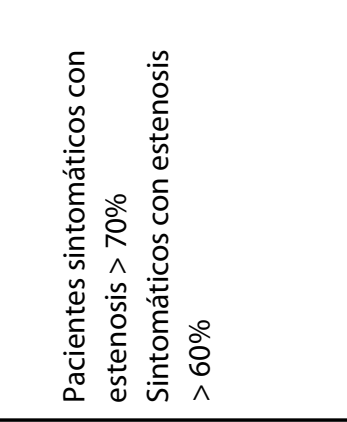 \\
\hline 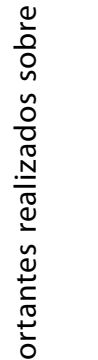 & 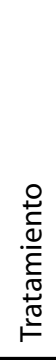 & 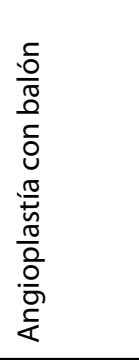 & 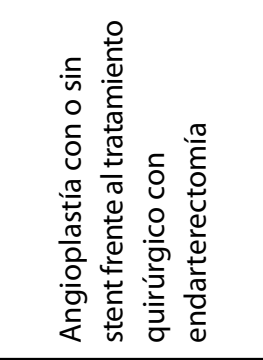 & 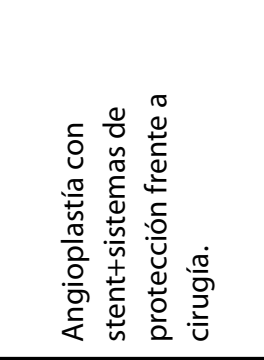 & 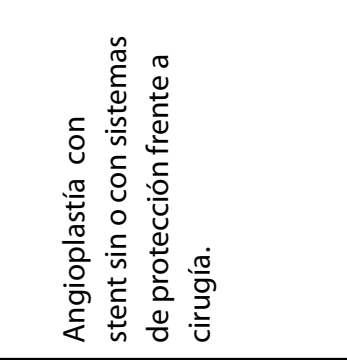 \\
\hline 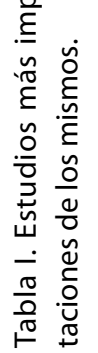 & 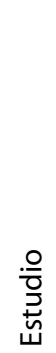 & 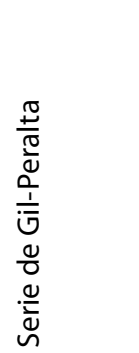 & 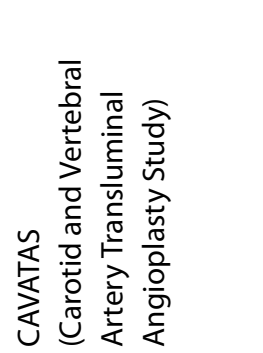 & 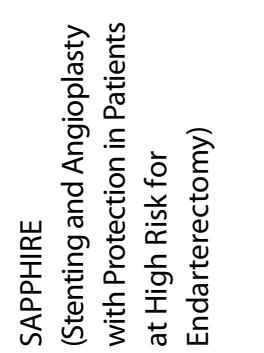 & 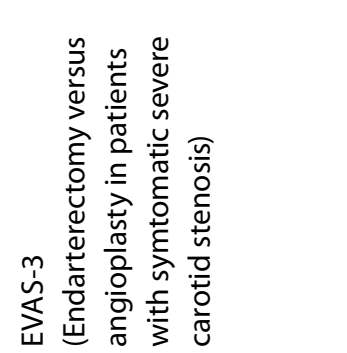 \\
\hline
\end{tabular}




\begin{tabular}{|c|c|c|c|c|}
\hline & 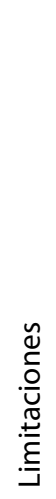 & 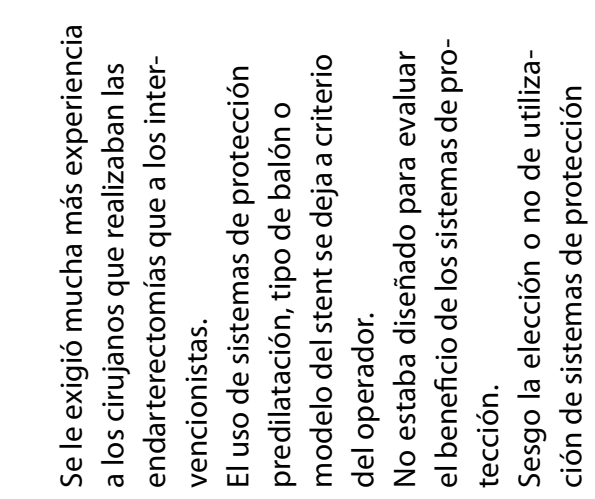 & & 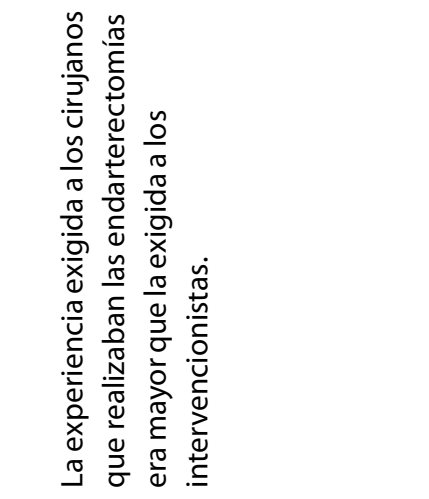 \\
\hline & 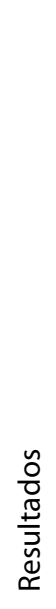 & 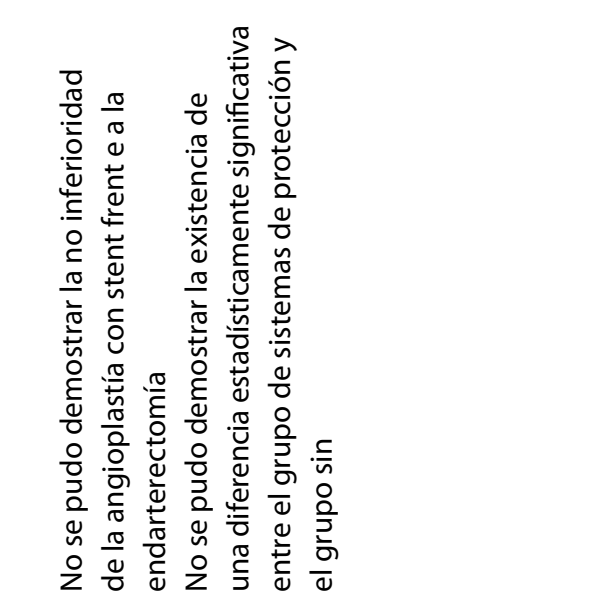 & 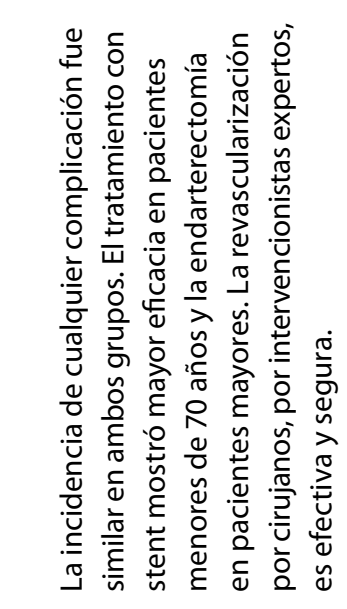 & 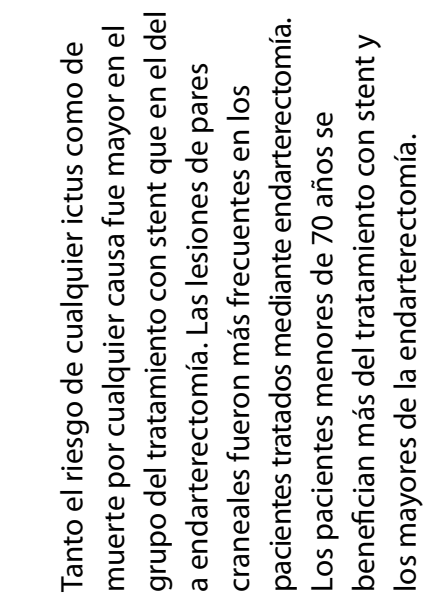 \\
\hline & 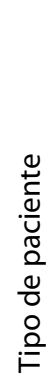 & 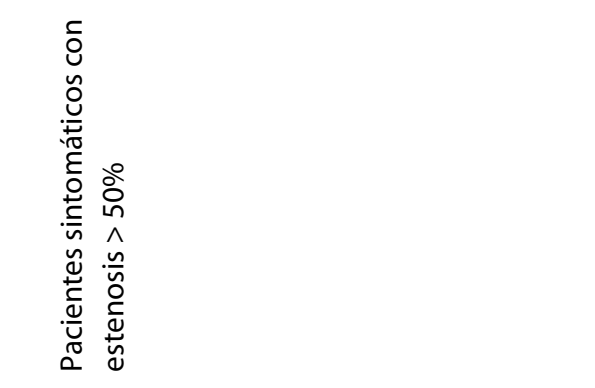 & 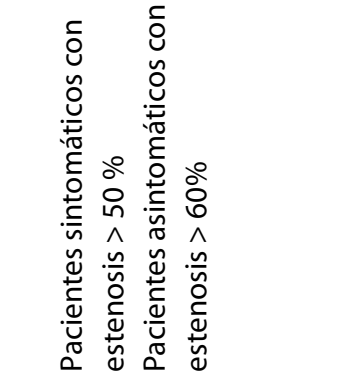 & 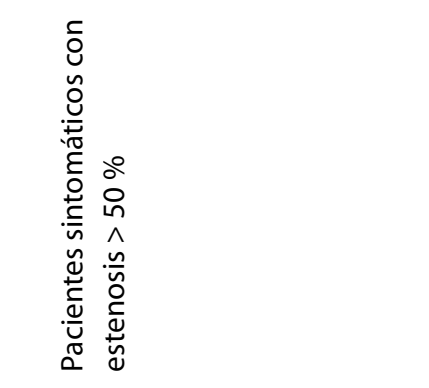 \\
\hline & 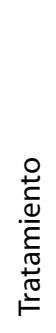 & 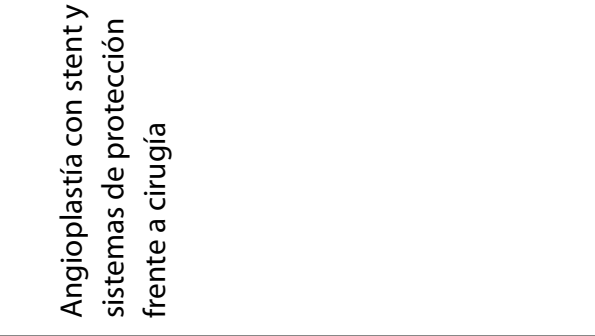 & 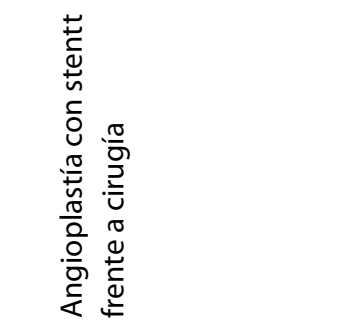 & 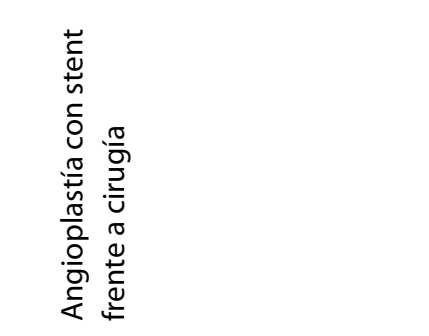 \\
\hline 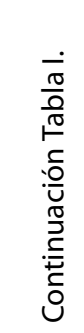 & 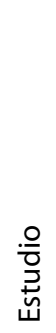 & 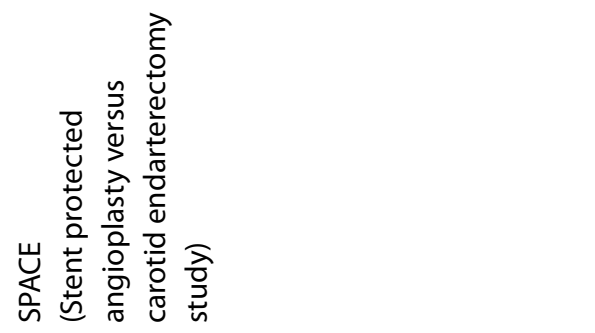 & 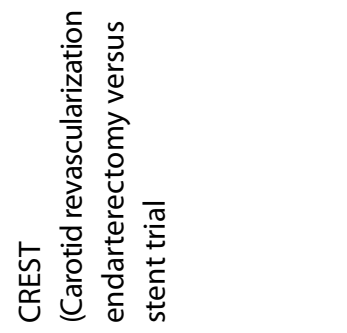 & 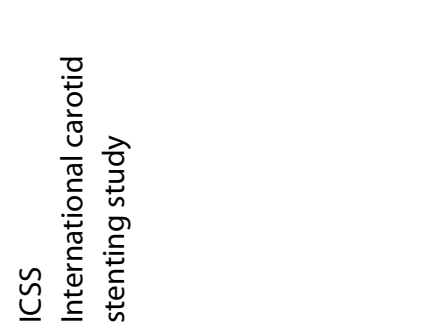 \\
\hline
\end{tabular}


y $9,9 \%$, respectivamente) ni en la eficacia a largo plazo. La lesión de pares craneales fue del 8,7\% en el grupo quirúrgico, mientras que la tasa de hematomas que requirieron cirugía fue del 6,7 y el $1,2 \%$ en el grupo de la endarterectomía y el endovascular, respectivamente. El $1 \%$ de los pacientes operados presentó un infarto agudo de miocardio. Sin embargo, la tasa de reestenosis fue superior en los pacientes sometidos a tratamiento endovascular (21\%) frente a la endarterectomía (5\%), sin asociación estadística entre reestenosis y recurrencia de síntomas. En todos los casos las reestenosis fueron asintomáticas. De los pacientes que se trataron con técnicas endovasculares, en la mayoría se realizó sólo angioplastía sin stent (80\%), y no se emplearon sistemas de protección.

En el seguimiento a 8 años, se observó una mayor incidencia de ACVA en el grupo endovascular, aunque las diferencias no fueron significativas entre ambos. A largo plazo se sigue observando una mayor tasa de reestenosis en el grupo tratado endovascularmente.

Stenting and Angioplasty with Protection in Patients at High Risk for Endarterectomy (SAPPHIRE) ${ }^{(17)}$ : Este estudio incluyó a 334 pacientes, de los cuales el 32\% presentaban una estenosis sintomática de un $50 \%$ o más y el $68 \%$ presentaba una estenosis asintomática de un $80 \%$ o mayor, medidas mediante Doppler. Se utilizaron sistemas de protección e implantación de stents autoexpandibles. Estos enfermos presentaban al menos, un factor de alto riesgo para endarterectomía (Tabla II). Se incluyó el desarrollo de infarto de miocardio como variable de análisis primaria acontecida a los 30 días y al año, en relación con el incremento de riesgo de muerte y de reinfarto que en los siguientes 6 meses presentan estos pacientes. La morbimortalidad a 30 días (muerte, ictus e infarto de miocardio) fue del $4,4 \%$ en el grupo de tratamiento con stent y del 9,9\% en el grupo quirúrgico. Los resultados a un año mostraron una diferencia estadísticamente significativa a favor de la angioplastía con stent, con una morbimortalidad del $12,2 \%$ frente a un $20,1 \%$ en el grupo de cirugía ${ }^{(18)}$. El SAPPHIRE se detuvo antes por no alcanzar el tamaño de muestra necesario para realizar el análisis estadístico.

Endarterectomy versus angioplasty in patients with symtomatic severe carotid stenosis (EVA-3S) ${ }^{(19)}$ : Es un estudio de no inferioridad, prospectivo, multicéntrico y ciego que valoró la seguridad y eficacia de la angioplastía carotidea con stent con o sin protección, frente al tratamiento mediante endarterectomía. Se incluyeron pacientes con estenosis carotidea sintomática del $70 \%$ o mayor. Posteriormente se incluyeron también pacientes sintomáticos con estenosis igual o mayor al $60 \%$.

El objetivo primario era evaluar si la angioplastía con stent, tanto sin como con sistema de protección
Tabla II. Muestra los criterios de alto riesgo quirúrgico para endarterectomía.

Criterios de alto riesgo para endarterectomía

- Insuficiencia cardiaca congestiva grados III/IV

- Fracción de eyección $<30 \%$

- Infarto agudo de miocardio reciente

- Angina inestable

- Necesidad de cirugía cardíaca en seis semanas

- Oclusión carotidea contralateral

- Parálisis laríngea contralateral

- Reestenosis post-endarterectomia previa

- Radioterapia cervical

- EPOC grave

- Más de 80 años

- Estenosis grave en tándem

es tan segura y efectiva como la cirugía carotidea en cuanto a la ocurrencia de ictus y/o muerte en los primeros 30 días postprocedimiento y a largo plazo.

El único estudio que había hasta la fecha con el que comparar era el CAVATAS. En él, la mayoría de los pacientes a los que se les realizó tratamiento endovascular no se les colocó stent y no se utilizaron sistemas de protección. La tasa de éxito en el CAVATAS fue del $89 \%$, que era similar a la obtenida en este estudio (92,5\%).

Comparando con el estudio SAPPHIRE, la incidencia de ictus en el grupo tratado con stent es mucho mayor en el EVA-3S. En él, el riesgo de cualquier ictus en los primeros 30 días tras tratamiento con stent sin sistemas de protección era tres veces mayor que el de pacientes tratados utilizando sistemas de protección. El riesgo de ictus o muerte a 30 días tras el tratamiento endovascular tanto en pacientes sintomáticos como asintomáticos era del $1,8 \%$ en 896 pacientes tratados con sistema de protección y del 5,5\% en 2.537 pacientes tratados sin sistemas de protección. Ésto sugiere que la angioplastía con stent con sistemas de protección es más segura que la angioplastía sin sistemas de protección. Aunque estos hallazgos necesitaban ser confirmados y los límites de los intervalos de confianza no presentaban significancia estadística, el comité de seguridad recomendó no realizar más angioplastías sin sistema de protección ${ }^{(19)}$.

Existen algunos factores que podrían explicar parte de los resultados obtenidos y la necesidad de detener el estudio antes de su finalización. El primero es que este ensayo no estaba diseñado para comparar los resultados del stent con protección frente a stent sin protección. El segundo es que la experiencia exigida a los cirujanos vasculares era mucho mayor que para los intervencionistas ${ }^{(7)}$. 
Stent protected angioplasty versus carotid endarterectomy study (SPACE) ${ }^{(20-22)}$ : Es un estudio prospectivo, multicéntrico y randomizado que se diseñó para demostrar la no inferioridad del stent frente a la endarterectomía. Se incluyeron 1.183 pacientes con estenosis carotídea sintomática del $50 \%$ o mayor, medido con criterios NASCET en angiografía, o del $70 \%$ o mayor si se medía mediante Doppler. Igual que en el EVAS-3, a los cirujanos se les exigía más experiencia que a los intervencionistas y el uso de sistemas de protección, la necesidad de predilatación, el tipo de balón o el modelo del stent se deja a criterio del operador.

El objetivo primario era medir la incidencia de ictus carotideo ipsilateral con síntomas de más de 24 horas de duración o muerte a los 30 días desde el momento de la randomización. No se pudo demostrar la no inferioridad de la angioplastía con stent frente a la endarterectomía en cuanto a la tasa de complicaciones a los 30 días, pero sí se hallaron diferencias en cuanto a la edad y el sexo. Se encontraron peores resultados para el tratamiento con angioplastía en los pacientes mayores de 75 años y mujeres.

Comparando con los ensayos previamente expuestos, el riesgo periprocedimiento en el tratamiento endovascular en el SPACE es parecido al de los grandes ensayos aleatorizados.

Un metaanálisis de los cinco estudios aleatorizados $^{(23)}$ reveló una tasa de complicaciones periprocedimiento de $8,1 \%$ en los pacientes del grupo del tratamiento endovascular y del $6,3 \%$ en los del grupo de la cirugía.

Este metaanálisis incluía los resultados del SAPPHIRE, el cual añadió heterogeneidad a estos datos, ya que incluía pacientes asintomáticos y con alto riesgo cardiovascular. En el SPACE, a los dos años del tratamiento, la incidencia de ictus ipsilateral fue igual en ambos grupos. Sin embargo, las reestenosis son significativamente más frecuentes en el grupo de tratamiento endovascular. Aunque no se puede excluir que el grado de estenosis intrastent se sobreestime debido a que se han utilizado los criterios convencionales de ecografía Doppler ${ }^{(21)}$.

Existe un subanálisis del SPACE que compara stent con sistemas de protección frente a stent sin sistemas de protección ${ }^{(18,22,24-26)}$.

El objetivo era medir la incidencia de ictus ipsilateral - muerte a 30 días en grupos de pacientes tratado sin o con sistemas de protección. Como el SPACE no estaba diseñado para evaluar el beneficio de los sistemas de protección, se estudió el efecto de los sistemas de protección como análisis secundario. Se analizaron los resultados en función del tipo de stent (celda abierta o cerrada) y de la utilización o no de filtros ${ }^{(27)}$, existiendo un sesgo en cuanto a la elección de utilización de sistemas de protección. La mayoría de stents de celda cerrada se implantaron sin sistemas de protección mientras que la mayoría de stents de celda abierta se implantaron con sistema de protección. En el grupo de celda cerrada, el uso de los filtros produjo un aumento en la tasa de complicaciones, sin embargo, en el grupo de celda abierta los sistemas de protección mostraron beneficio. Por la falta de aleatorización de los pacientes, no se pudo demostrar una diferencia estadísticamente significativa entre ambos grupos, por lo que se pone en duda la utilidad de los sistemas de protección.

En el metaanálisis realizado entre el EVAS-3 y el SPACE igualmente no se pudo demostrar la existencia de diferencias entre las dos formas de tratamiento ${ }^{(28,29)}$.

A la vez han surgido estudios independientes que tampoco han apoyado la utilización de sistemas de protección. Técnicamente, estos sistemas pueden reducir, pero no eliminar la suelta de émbolos ${ }^{(39)}$ ya que hay que tener en cuenta que su propio uso puede provocar vasoespasmo o disecciones ${ }^{(31)}$.

Los sistemas de protección pueden ser beneficiosos cuando se utilizan stents de celda abierta, pero no lo son tanto cuando se implantan stents de celda cerrada ${ }^{(27)}$.

Carotid revascularization endarterectomy versus stent trial (CREST) ${ }^{(32)}$ : Es un estudio randomizado a doble ciego que comparó endarterectomía con angioplastía con stent. Ambos incluían pacientes sintomáticos y asintomáticos con estenosis carotidea, medida en angiografía, de más del $50 \%$ o del $60 \%$ respectivamente. El objetivo primario era determinar la tasa de ictus, infarto agudo de miocardio o muerte periprocedimiento (30 días desde el momento de la aleatorización) y la tasa de ictus ipsilateral a largo plazo (4 años desde la randomización).

Durante los primeros 30 días postrandomización, la incidencia de cualquier complicación fue similar en ambos grupos (5,2\% y 4,5\% respectivamente). Aunque sí se encontraron diferencias entre las distintas complicaciones.

A los cuatro años no se observaron diferencias significativas entre ambos grupos. La tasa de cualquier complicación fue de $7,2 \%$ en el grupo de tratamiento endovascular y de $6,8 \%$ en el grupo de endarterectomía. La tasa de ictus o muerte fue del $6,4 \%$ en el grupo de angioplastía y del $4,7 \%$ en de la endarterectomía. Al igual que en SPACE y ICCS (del que se hablará a continuación), sí se observó relación entre la edad y la eficacia del tratamiento. El stent mostró mayor eficacia en pacientes menores de 70 años y la endarterectomía en pacientes mayores de 70 . Esta misma relación coincide con el estudio SPACE y con el ICSS (que se expondrá a continuación).

Tampoco se observaron diferencias entre pacientes sintomáticos y asintomáticos ni entre sexos.

International carotid stenting study $(\mathrm{ICSS})^{(33,34)}$ : El objetivo de este estudio aleatorizado era comparar el riesgo-beneficio y coste-efectividad del tratamiento 
mediante angioplastía con stent, de estenosis carotidea en pacientes sintomáticos, comparado con el tratamiento mediante endarterectomía. El objetivo primario era establecer si existían diferencias significativas en cuanto a la tasa a largo plazo de muerte o ictus discapacitante, entre pacientes tratados con endarterectomía y tratados con stents. Una vez más, la experiencia exigida a los cirujanos era mucho mayor que la exigida a los intervencionistas. En este estudio se introdujo como novedad la utilización protocolizada de los sistemas de protección siempre que su uso no fuera más arriesgado que el no utilizarlo.

Los pacientes se siguieron a los 30 días, postrandomización, a los 6 meses y al año hasta los cinco años. Como objetivos secundarios se determinron la diferencia entre ambos grupos con respecto a la tasa de cualquier ictus, infarto de miocardio, o muerte a los 30 días. Incluso se analizaron las tasas de reestenosis y la calidad de vida.

A los 120 días tras la aleatorización, se observaron 34 ictus discapacitantes o muertes en el grupo del tratamiento con stent, comparados con 27 eventos en el grupo de la endarterectomía. La incidencia de ictus, muerte o infarto de miocardio fue del 8,5\% en el grupo de stents y del 5,2\% en el grupo de la endarterectomía. Tanto el riesgo de cualquier ictus como de muerte por cualquier causa fue mayor en el grupo del tratamiento con stent que en el de la endarterectomía. Por el contrario las lesiones de pares craneales fueron mayores en los pacientes tratados mediante endarterectomía. Igualmente se observó menor tasa de hematomas en el grupo del tratamiento con stent.

No se observaron diferencias entre sexos, pero sí en cuanto a la edad. Los pacientes menores de 70 años se benefician más del tratamiento con stent.

Estos autores concluyen que la endarterectomía debería ser el tratamiento de elección para la estenosis carotidea sintomática candidatos a cirugía. No obstante, aquellos con contraindicación de cirugía serían candidatos a colocación de stent.

Existen resultados dispares en los diversos estudios bien por heterogeneidad en las poblaciones, la experiencia de los operadores o en el material utilizado. Ésto dificulta el establecimiento de conclusiones. Múltiples estudios coinciden en que el tratamiento con stent beneficia más a pacientes menores de 70 años y presenta al menos iguales resultados que la endarterectomía a corto plazo, con una mayor tasa de reestenosis a largo plazo. Pero no se ha podido establecer todavía cuál es el mejor tratamiento ni cuáles son los criterios que deben tenerse en cuanta a la hora de elegir uno u otro tratamiento (Figura 1).

\section{Endarterectomía vs tratamiento endovascular.}

De acuerdo con lo establecido por el grupo español de neurorradiología intervencionista $(\mathrm{GENI})^{(7)}$ las indi-

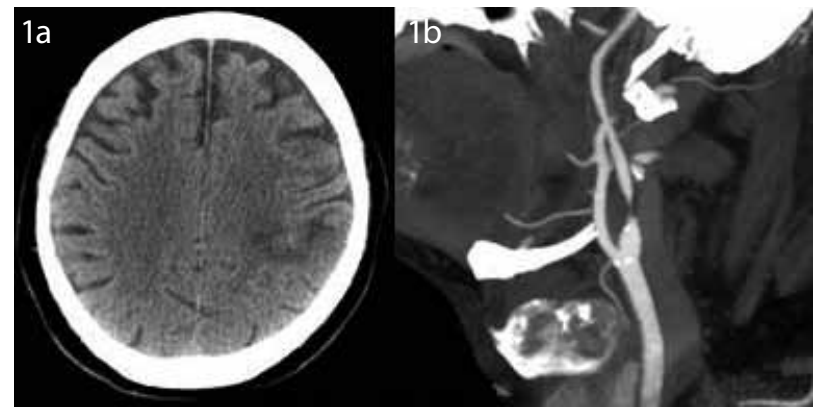

Figura 1. Paciente de 75 años con cuadro un mes atrás, de hemiparesia derecha tras varios episodios de accidentes isquémicos transitorios a pesar de estar doblemente antiagregado. a) tomografía cerebral durante el ingreso. Se observan signos de infarto en territorio de arteria cerebral media izquierda. b) TC angiografía de troncos supra-aórticos. Se visualiza una estenosis significativa en el origen de la carótida interna izquierda.

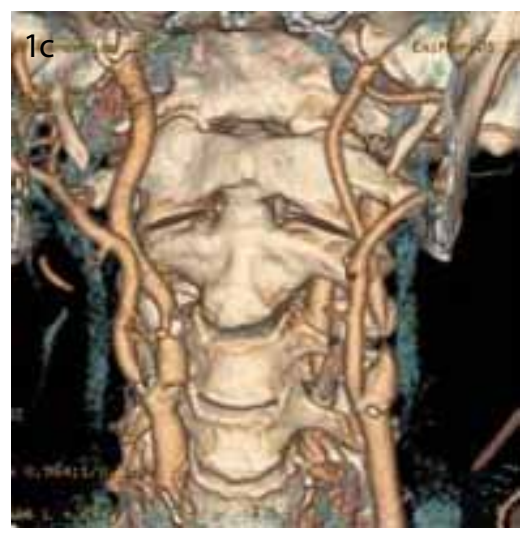

Figura 1c. Reconstrucciones tridimensionales del TC descrito en el apartado anterior.

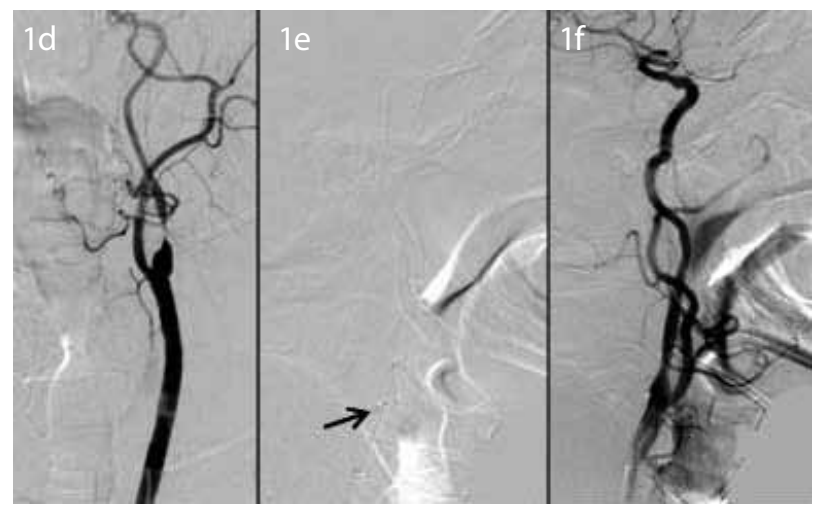

Figura 1d. Angiografía en plano oblicuo de carótida izquierda. Se confirma la estenosis descrita en el TC. e) Tratamiento endovascular. La microguía se coloca distalmente en carótida cavernosa y se despliega el stent (flecha). f)Control angiográfico tras la colocación del stent. No se observa estenosis residual. 
caciones de tratamiento en la estenosis de carótida, deberían establecerse de forma consensuada en equipos multidisciplinarios. En este ámbito, se deben desarrollar protocolos para la selección, exclusión o inclusión.

Las ventajas y limitaciones del tratamiento endovascular se reflejan en la Tabla III.

En la práctica, el tratamiento endovascular de la estenosis de carótida se indicaría en aquellos pacientes que se beneficiarían de una endarterectomía, pero en los que la cirugía no se considere adecuada, por presentar un alto riesgo quirúrgico/anestésico.

Otros ensayos clínicos que comparan angioplastía y endarterectomía están en marcha, como el ACT1, que evaluará pacientes sintomáticos de bajo riesgo quirúrgico y el Transatlantic Asymptomatic Carotid InterventionTrial (TACIT), que reclutará pacientes asintomáticos para ser aleatorizados en tres grupos: angioplastía, endarterectomía y mejor tratamiento médico. Este tercer grupo es importante dado que el mejor tratamiento médico actualmente difiere del aplicado en los ensayos clínicos de referencia que datan de más de una década ${ }^{(35)}$ y existen evidencias de que su impacto sobre la enfermedad es más favorable de lo anteriormente considerado lo que podría restar margen de beneficio tanto al tratamiento quirúrgico como al endovascular ${ }^{(36)}$.
Conclusiones

Existen resultados dispares en los diversos estudios bien sea por heterogeneidad en las poblaciones, la experiencia de los operadores, el material o la técnica empleada y las variables tenidas en cuenta. Igualmente algunos de los estudios se llevaron a cabo hace décadas, por lo que tanto la técnica endovascular como los tratamientos médicos empleados entonces, no son comparables con los empleados hoy en día. Esto hace difícil el establecimiento de conclusiones para decidir cuál es el tratamiento óptimo en cada caso. Queda claro, en los diversos ensayos, que el tratamiento con stent beneficia más a pacientes menores de 70 años y presenta al menos iguales resultados que la endarterectomía a corto plazo, con una mayor tasa de reestenosis a largo plazo; pero aún quedan por resolver la indicación de tratamiento en pacientes asintomáticos o la utilización o no de sistemas de protección. Habrá que esperar los resultados de los nuevos ensayos que se están llevando a cabo. En lo que no hay disparidad es que el establecimiento del tratamiento debe determinarse de manera individualizada para cada paciente y por un equipo multidisciplinar.

Tabla III. Ventajas y limitaciones del tratamiento endovascular

Ventajas de tratamiento endovascular frente a la cirugía

Limitaciones del tratamiento endovascular

- Ausencia de incisión cervical

- Ausencia de lesiones de pares craneales

- Mejor acceso a lesiones altas

- Posibilidad de tratar lesiones en tándem y

la enfermedad multivaso

- No necesidad de anestesia general

- Mejor control clínico de complicaciones

- Menor tiempo de isquemia cerebral

- Menor tiempo de convalecencia.

\section{Bibliografía}

1. Pérez P, Martínez J, Ruiz M, Blázquez JA, del Llano JE. Evaluación de la estenosis de la arteria carótida interna por angiografía RM con contraste: revisión sistemática de la bibliografía. Radiología. 2004; 46: 20-8

2. Santos AL, Ramos M, Delgado F, Cano A, Bravo F. Angio-TC en la evaluación de estenosis de la bifurcación arterial carotídea: comparación con arteriografía por sustracción digital intraarterial Radiología 2001; 43(6): 273-278.

3. Field TS, Benavente OR. Current status of antiplatelet agents to prevent stroke. Curr Neurol Neurosci Rep. 2011; 11(1): 6-14.

4. Diener et al. European stroke prevention study 2 . Dipiridamole and acetylsalicylic acid in the secondary prevention of stroke. Journal of neurological sciences 1996; 143: 1-13.

5. North American Symptomatic Carotid Endarterectomy Trial Collaborators. Beneficial effect of carotid endar- terectomy in symptomatic patients with high-grade carotid stenosis. N Engl J Med 1991; 325: 445-453.

6. European Carotid Surgery Trialist's Collaborative Group: Randomised trial of endarterectomy for recently symptomatic carotid stenosis: final results of the MRC European Carotid Surgery Trial (ECST). Lancet 1998; 351:1379-1387

7. E. Castro-Reyes, A. González-García, en representación del Grupo Español de Neurorradiología Intervencionista (GENI). Tratamiento endovascular de la enfermedad carotídea. Situación actual, aspectos técnicos y capacitación profesional Rev Neurol 2005; 41(12): 749-756

8. Kerber CW, Hornwell LD, Loeden OL. Catheter dilatation of proximal carotid stenosis during distal bifurcation endarterectomy. AJNR Am J Neuroradiol 1980; 1: 348-349.

9. Gil-Peralta A, Mayol A, Marcos JR, Gonzalez A, Ruano J, Boza F, Duran F. Percutaneous transluminal angioplasty of the symptomatic atherosclerotic carotid 
arteries. Results, complications, and follow-up. Stroke. 1996; 27(12): 2271-2273.

10. Frericks H, Kievit J, van Baalen JM, van Bockel JH. Carotid recurrent stenosis and risk of ipsilateral stroke: a systematic review of the literature. Stroke 1998; 29: 244-250.

11. Moore WS, Kempczinski RF, Nelson JJ, Toole JF. Recurrent carotid stenosis: results of the asymptomatic carotid atherosclerosis study. Stroke 1998; 29: 20182025.

12. Markus HS, Clifton A, Buckenham T, Brown MM. Carotid angioplasty: detection of embolic signals during and after the procedure. Stroke 1994; 25: 2403-2406.

13. Crawley F, Clifton A, Buckenham T, Loosemore T, Taylor RS, Brown MM. Comparison of hemodynamic cerebral ischemia and microembolic signals detected during carotid endarterectomy and carotid angioplasty. Stroke 1997; 28: 2460-2464.

14. McCleary AJ, Nelson M, Dearden NM, Calvey TA, Gough MJ. cerebral haemodynamics and embolization during carotid angioplasty in high-risk patients. Br J Surg 1998; 85: 771-774.

15. Constantine C. Phatouros, Randall T. Higashida. Carotid Artery Stent Placement for Atherosclerotic Disease: Rationale, Technique, and Current Status. Radiology 2000; 217: 26-41.

16. CAVATAS investigators. Endovascular versus surgical treatment in patients with carotid stenosis in the Carotid and Vertebral Artery Transluminal angioplasty Study (CAVATAS): a randomised trial. Lancet 2001; 357: 1729-1737.

17. Yadav JS, Wholey MH, Kuntz RE, Fayad P, Katzen BT, Mishkel GJ. Stenting and Angioplasty with Protection in Patients at High Risk for Endarterectomy Investigators. Protected carotid-artery stenting versus endarterectomy in high-risk patients. N Engl J Med. 2004; 351(15): 493501.

18. Kastrup A, Gröschel K, Krapf H, Brehm B, Dichganj J, Schulz J. Early outcome of carotid angioplasty and stenting with and without cerebral protection devices: a systematic review of the literature. Stroke 2003; 34: 813-9.

19. Trial EVA-3S Investigators. Carotid Angioplasty and Stenting With and Without Cerebral Protection Clinical Alert From the Endarterectomy Versus Angioplasty in Patients With Symptomatic Severe Carotid Stenosis . Stroke. 2004; 35: e18-e21.

20. Wiesmann M, Schöpf V, Jansen O, Brückmann H. Stentprotected angioplasty versus carotid endarterectomy in patients with carotid artery stenosis: meta-analysis of randomized trial data. Eur Radiol. 2008; 18(12): 2956-2966.

21. Eckstein $\mathrm{HH}$, Ringleb $\mathrm{P}$, Allenberg JR, Berger J, Fraedrich G, Hacke W et al. Results of the Stent-Protected Angioplasty versus Carotid Endarterectomy (SPACE) study to treat symptomatic stenoses at 2 years: a multinational, prospective, randomised trial. Lancet Neurol. 2008; 7(10): 893-902. Erratum in: Lancet Neurol. 2009; 8(2): 135.

22. SPACE Collaborative Group, Ringleb PA, Allenberg J, Brückmann $\mathrm{H}$, Eckstein HH, Fraedrich G, Hartmann $M$ et al.30 day results from the SPACE trial of stentprotected angioplasty versus carotid endarterectomy in symptomatic patients: a randomised non-inferiority trial. Lancet. 2006; 368(9543): 1239-1247. Erratum in: Lancet. 2006; 368(9543): 1238.

23. Coward LJ, Featherstone RL, Brown MM. Safety and efficacy of endovascular treatment of carotid artery stenosis compared with carotid endarterectomy: a Cochrane systematic review of the randomized evidence. Stroke 2005; 36: 905-911.

24. Reimers B, Corvaja N, Moshiri S, Sacca` S, Albiero R, Di Mario $C$ et al. Cerebral protection with filter devices during carotid artery stenting. Circulation. 2001; 104: 12-15.

25. Castriota F, Cremonesi A, Manetti R, Liso A, Oshoala $\mathrm{K}$, Ricci $\mathrm{E}$ et al. Impact of cerebral protection devices on early outcome of carotid stenting. J Endovasc Ther. 2002; 9: 786-792.

26. Cremonesi A, Manetti R, Castriota F, Setacci F, Setacci $C$. Protected carotid stenting: clinical advantages and complications of embolic protection devices in 442 consecutive patients. Stroke. 2003; 34: 1936-1943.

27. Olav Jansen, Jens Fiehler, Marius Hartmann, Hartmut Bruckmann. Protection or Nonprotection in Carotid Stent Angioplasty. The Influence of Interventional Techniques on Outcome Data From the SPACE Trial. Stroke. 2009; 40: 841-846.

28. Duda Sh, Wiskirchen J, Tepe G, Bitzen M, Kaulich Tw, Stoeckel D et al. Physical properties of endovascular stents: an experimental comparison. J Vasc Interv Radiol. 2000; 11: 645-654.

29. Bosiers M, Deloose K, Verbist J, Peeters P. Carotid artery stenting: which stent for which lesion? Vascular. 2005; 13: 205-210.

30. Orlandi G, Fanucchi S, Fioretti C, Acerbi G, Puglioli M, Padolecchia R et al. Characteristics of cerebral microembolism during carotid stenting and angioplasty alone. Arch Neurol. 2001; 58: 1410-1413.

31. Eckert B, Zeumer H. Carotid artery stenting with or without protection devices? Strong opinions, poor evidence! Stroke. 2003; 34: 1941-1943.

32. Brott TG, Hobson RW, Howard G, Roubin GS, Clark WM, Brooks W, et al; CREST Investigators. Stenting versus endarterectomy for treatment of carotid-artery stenosis. N Engl J Med. 2010; 363(1): 11-23.

33. Featherstone RL, Brown MM, Coward LJ; ICSS Investigators. International carotid stenting study: protocol for a randomised clinical trial comparing carotid stenting with endarterectomy in symptomatic carotid artery stenosis. Cerebrovasc Dis. 2004; 18(1): 69-74.

34. International Carotid Stenting Study investigators, Ederle J, Dobson J, Featherstone RL, Bonati LH, van der Worp HB, de Borst GJ, et al. Carotid artery stenting compared with endarterectomy in patients with symptomatic carotid stenosis (International Carotid Stenting Study): an interim analysis of a randomised controlled trial. Lancet. 2010; 375(9719): 985-997.

35. Alcazar PP, Garcia BE, Fandiño BE. Avances en neurorradiología intervencionista. Radiología. 2010; 52(Supl.2): 46-55.

36. Marquardt L, Geraghty OC, Mehta Z, Rothwell PM, Low Risk of Ipsilateral Stroke in Patients With Asymptomatic Carotid Stenosis on Best Medical Treatment. A Prospective ,Population-Based Study. Stroke. 2010; 41: e11-17. 